\title{
Radiology
}

\section{A Case Report of Perineural Cysts, Also Known as Tarlov Cysts}

\author{
Dijana Zadravec, MD, $\mathrm{PhD}^{1,2}$; Petra Margetic, $\mathrm{MD}, \mathrm{PhD}^{1,2^{*}}$; \\ Mia Smoljan, $\mathrm{MD}^{1,2}$; Ivana Zupetic, $\mathrm{MD}^{1}$ \\ ${ }^{1}$ University Hospital Center Sestre milosrdnice \\ ${ }^{2} S c h o o l$ of Dental Medicine, University of Zagreb \\ Zagreb, Croatia
}

\begin{abstract}
Tarlov cysts (TCs) are a rare but intensive cause of chronic low back pain or lumbosacral radiculopathy and should be considered in the differential diagnosis of radicular symptoms. Magnetic resonance imaging (MRI) is the radiologic study of choice to visualize these cysts.

In the current study, we describe a case of a 49-year-old patient with intermittent pain on his coccigeal region, right buttock, and intensive pain in the right hip and inner side of the thigh, in 1.5 years of duration. MRI of the lumbosacral spine and hip showed right-sided, sacral S2 and S3 perineural cysts, the largest located at the right S2 nerve root. Conservative treatment of sacral perineurial cysts should be the first choice. The surgical treatment is recomended in cases when MRI scans reveal a large $(1.5 \mathrm{~cm})$ cyst associated with neurological symptoms and no response on conservative treatment.(International Journal of Biomedicine. 2019;9(1):66-68.)
\end{abstract}

Key Words: meningeal cyst $\bullet$ Tarlov cyst $\bullet$ CT myelography $\bullet$ MRI $\bullet$ neurological symptoms

\section{Introduction}

Spinal meningeal cysts have been classified by Nabors et al. ${ }^{(1)}$ into three different types:

- Type I: Extradural meningeal cysts without spinal nerve root fibers

- Type II: Extradural meningeal cysts with spinal nerve root fibers (TCs)

- Type III: Spinal intradural meningeal cysts

Perineural (or Tarlov) cysts (TCs) are defined as cerebrospinal fluid (CSF)-filled saccular lesions located in the extradural space of the spinal canal and formed within the nerve root sheath of the dorsal root ganglion (between the peri- and endoneural spaces of the spinal posterior nerve root sheath). This saccular dilatation of the spinal posterior nerve root sheath has microconnections to the subarachnoid space.

Thus, when pulsatile and hydrodynamic forces of CSF, through a ball-valve mechanism and gravitational pressure within the cyst, cause these perineural cysts to fill and

*Corresponding author: Petra Margetic, MD, PhD. RTG department, University Hospital Center Sestre milosrdnice. Zagreb, Croatia.E-mail: hpetra23@gmail.com expand in size, it can cause neural compression, resulting in neurological symptoms. ${ }^{(2)}$

Perineural cysts typically exhibit delayed filling with a contrast medium on myelography (Figures 1 and 2), which is one of the criteria used by Tarlov to distinguish PCs from meningeal diverticula.
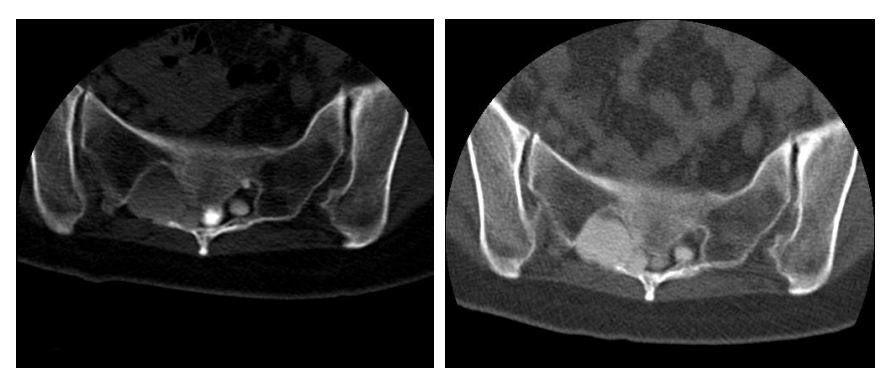

Fig. 1. CT myelography: early Fig. 2. CT myelography: late postpostcontrast image. contrast image.

The incidence of TCs in the adult population is between $4.6 \%$ and $9 \%$. The incidence does not significantly differ between sexes but is more prevalent in younger people: $4.0 \%$ in people less than 50 years of age versus $1.3 \%$ in people over 50 years. ${ }^{(3)}$ 
On postmyelographic computed tomography immediately after contrast medium injection, the largest cyst at right S2 showed selective filling of the contrast medium, suggesting that inflow of cerebrospinal fluid to the cyst exceeded outflow. Six hours after the injection, the intensity of the cysts was similar to the intensity of the thecal sac.

\section{Case presentation}

A 49-year-old patient presented with intermittent pain on his coccigeal region, right buttock, and intensive pain in the right hip and inner side of the thigh, in 1.5 years of duration. Local pain is caused by cyst enlargement and by pressuring the periost or joint capsule. Radiculopathy results from a cyst compressing the nerve root, and in the case of ventral root compression, motor weakness occurs.

Patient reported exacerbation of symptoms when standing, coughing and lifting. Later the patient started having rest pain as well. On clinical examination, distribution of the pain corresponded to the S2-S3 dermatome. Straight leg raising was $40^{\circ}$ on the right side and normal on the left side. There was significant blunting of sensations along the $\mathrm{S} 1$ and S2 dermatome on the right side, with a slight motor deficit in right lower limb. X-ray of the lumbosacral spine did not reveal any abnormality. Magnetic resonance imaging (MRI) of the lumbosacral spine and hip showed right-sided, sacral S2 and S3 perineural cysts, the largest located at the right S2 nerve root (Figures 3-6). Note the resulting denervation edema in the right gluteal muscle.

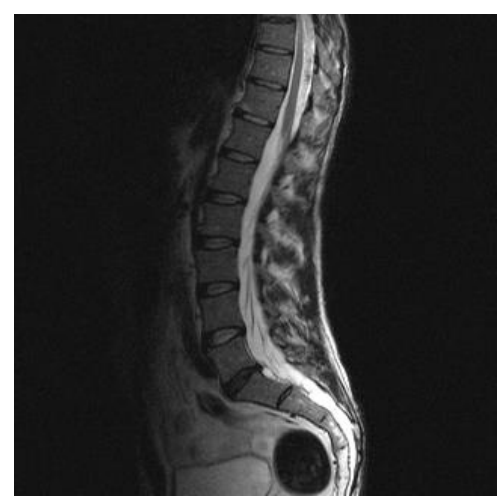

Fig. 3. MRI of the lumbosacral spine: sagital plane; right-sided sacral S2 and $S 3$ perineural cysts, the largest located at the right $S 2$ nerve root.

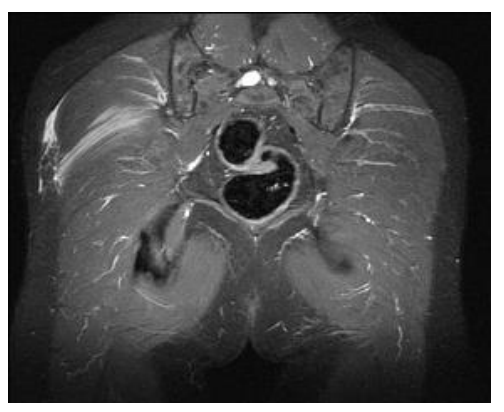

Fig. 4. MRI of the lumbosacral spine: transversal plane.

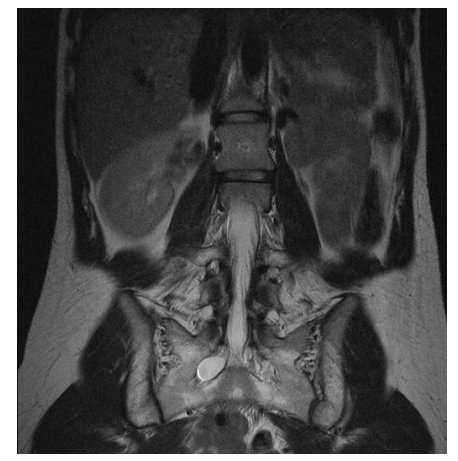

Fig. 5. MRI of the lumbosacral spine: coronal plane.

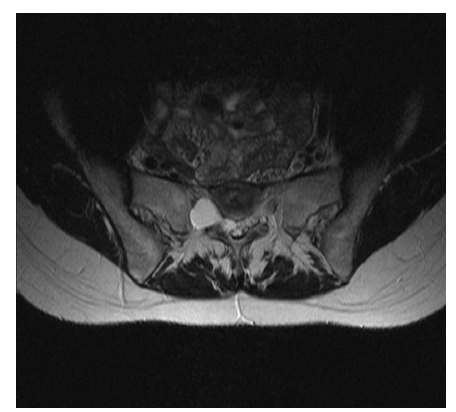

Fig. 6. MRI axial T2WI: a CSFfilled cystic lesion, $1.5-1.8 \mathrm{~cm}$ in diameter, sitting opposite $S 2$.

The patient was, after 8 months of conservative treatment, taken for a sacral laminectomy, excision of the cyst and plication of the cyst wall, while retaining the nerve root. Histopathological examination of the cyst wall showed nerve cells, which confirmed the diagnosis of a Tarlov cyst. Patient experienced relief of pain immediately after the surgery. On follow-up, the patient had no pain in lower limbs and back.

\section{Discussion}

Tarlov cysts are rare causes of low back pain, but when they are present pain is very intense, leading to disability of normal working and living activities.

Plain X-rays are usually normal. They may reveal characteristic bone erosion of the spinal canal or anterior or posterior neural foramina.

MRI gives better soft tissue contrast and is currently the investigation of choice for perineural cysts. The cysts demonstrate a low signal on T-1 weighted images and a high signal on T-2 weighted images, similar to CSF.

Myelography showing the filling of the meningocele sac $1 \mathrm{~h}$ after injection of the contrast medium is highly suggestive of a perineural Tarlov cyst.

A CT scan can demonstrate cystic masses isodense with CSF located at the foramina.

The pathogenesis of perineural cysts is uncertain. Tarlov felt that hemorrhage into the subarachnoid space caused accumulations of red cells, which impeded the drainage of the veins in the perineurium and epineurium, leading to rupture with subsequent cyst formation. There is no consensus on a single method of treatment. Various methods have been advocated. Tarlov advised that symptomatic, single perineural cysts should be completely excised together with the posterior root and ganglion from which they arise. ${ }^{(4)}$ 
According to Caspar, microsurgical excision of the cyst combined with duraplasty or plication of the cyst wall is an effective and safe treatment of symptomatic sacral cysts. The parent nerve root is always left intact. ${ }^{(5)}$

\section{Conclusion}

Tarlov cysts are rare, but they cause chronic low back pain or lumbosacral radiculopathy and should be considered in the differential diagnosis of radicular symptoms. MRI is the radiologic study of choice to visualize these cysts.

Conservative treatment of sacral perineurial cysts should be the first choice. The surgical treatment is recomended in cases when MRI scans reveal a large $(1.5 \mathrm{~cm})$ cyst associated with neurological symptoms and no response on conservative treatment.

\section{Conflict of Interest}

The authors declare that they have no competing interests.

\section{Acknowledgments}

The authors thank the surgeons of University Hospital for clinical support.

\section{References}

1. Nabors MW, Pait TG, Byrd EB, Karim NO, Davis DO, Kobrine AI, Rizzoli HV. Updated assessment and current classification of spinal meningeal cysts. J Neurosurg. 1988;68(3):366-77.

2. Mummaneni PV, Pitts LH, McCormack BM, Corroo JM, Weinstein PR. Microsurgical treatment of symptomatic sacral Tarlov cysts. Neurosurgery. 2000;47(1):74-8; discussion 78-9. 3. Park HJ, Jeon YH, Rho MH, Lee EJ, Park NH, Park SI, Jo JN. Incidental findings of the lumbar spine at MRI during herniated intervertebral disk disease evaluation. AJR Am J Roentgenol. 2011;196(5):1151-5.

4. Tarlov IM. Spinal perineurial and meningeal cysts. J Neural Neurosurg Psychiatry. 1970;33(6):833-43.

5. Caspar W, Papavero L, Nabhan A, Loew C, Ahlhelm F. Microsurgical excision of symptomatic sacral perineural cysts: A study of 15 cases. Surg Neurol. 2003;59(2):101-5; discussion 105-6. 